\title{
A MODEL FOR SYMMETRIES IN NONLINEAR TRANSPORT
}

\author{
R.F. RODRÍGUEZ ${ }^{\ddagger}$ and N.G. VAN KAMPEN \\ Instituut voor Theoretische Fysica der Rijksuniversiteit te Utrecht, \\ Utrecht, The Netherlands
}

Received 15 July 1975

A model for a Rayleigh particle suspended in a rarefied gas in internal equilibrium is constructed. It is shown that the macroscopic evolution of this system can be described by using nonlinear unilateral transfer flows which are gradients of particular scalar functions. These functions are constructed according to a general theory of nonlinear irreversible processes proposed previously by van Kampen.

\section{Introduction}

Many attempts have been made to generalize the Onsager theory of irreversible processes to the nonlinear case, in which the connection between the thermodynamic flows $J_{v}$ and the generalized forces $X_{v}$ should be given by nonlinear functions. Recently it has been suggested by several authors ${ }^{1-3}$ ) that the proper generalization of Onsager's reciprocity relations is of the form

$$
J_{v}=\frac{\partial K(X)}{\partial X_{v}}
$$

where $K(X)$ is a function of the generalized forces and has to be determined for the particular process being considered. Accordingly this function plays the essential role of a generating function of the net thermodynamic flows and thus determines the macroscopic evolution of the system. From the previous relation it follows that

$$
\frac{\partial J_{v}}{\partial X_{\mu}}=\frac{\partial J_{u}}{\partial X_{v}}
$$

₹ On leave of absence from the Facultad de Ciencias, Universidad Nacional Autónoma de México, México, D.F. 
and from this we recover the usual reciprocity relation between Onsager coefficients when the flows are linear functions of the forces.

It should be emphasized that the theories proposing relation (1) are phenomenological, they do not give, nor pretend to give, a detailed microscopic interpretation of the irreversible process or of the function $K$. For example, the general idea of Oster $e t$ al. ${ }^{1}$ ) is to generalize graph techniques used in network theory in order to obtain a formal framework for treating complex thermodynamical processes. A different general approach is followed by Edelen ${ }^{3}$ ), where the properties of a certain class of convex functionals of the thermodynamical state variables are used to yield reciprocity relations of the general form (2). On the other hand, at present, a rigorous proof of what the nonlinear reciprocity relations should be, based on general statistical mechanical arguments, has not been given. And of course, there does not exist a nonequilibrium formalism analogous to the partition sum algorithm of equilibrium statistical mechanics, which provides a systematic method for the calculation of any macroscopically observable property of the system undergoing an arbitrary irreversible process. The theory proposed by van Kampen $^{4}$ ) is also phenomenological, but nevertheless, it establishes a connection between the microscopic elementary events and the macroscopic evolution of the system. For future reference we recall briefly the principal assumptions and consequences of this theory.

It is assumed that the closed system $\mathscr{S}$ undergoing an irreversible process can be decomposed into subsystems $\mathscr{S}^{r}$ which are in equilibrium, although $\mathscr{S}$ is not necessarily in equilibrium. The contact between any pair of subsystems allows transfers of certain physical quantities $A_{v}$ (mass, energy, momentum, electric charge, etc.) from one to the other, with the effect of producing an increase of the total entropy. It is considered that these transfers are due to microscopic events, which originating in one subsystem carry definite amounts of the $A_{v}$ 's to the other system. The probability of occurrence of such an elementary event is supposed to depend only on the thermodynamic state of the source system and on the nature of the contact with the receiving system, but not on its state. As a result of these assumptions the evolution of $\mathscr{S}$ can be decomposed into a combination of unilateral transfer flows $F_{v}$, and the phenomenological equations of $\mathscr{S}$ are of the form

$$
\dot{a}_{v}^{r s}=J_{v}^{r s}=\sum_{r}\left\{F_{v}^{r s}\left(X^{s}\right)-F_{v}^{s r}\left(X^{r}\right)\right\} .
$$

This visualization of the irreversible process as a sequence of elementary events leads to a very important relation. An elementary event only takes place when the molecules involved in it are in a proper region of the phase space of the subsystem $\mathscr{S}^{s}$. As a result, the molecules of $\mathscr{S}^{s}$ are divided into two groups: the active and the remaining particles, and the probability that such a division takes place is proportional to the fraction of phase space of $\mathscr{S}^{s}$ in which this decomposition is realized. This is really a generalized form of the classical Stoßzahlansatz and 
makes it possible to find a scalar function $K\left(X^{s}\right)$ of the forces, the first derivatives of which yield the unilateral flows appearing in the phenomenological equations (3):

$$
\Gamma_{v}^{r s}\left(X^{s}\right)=-\frac{\partial K^{r s}(X)}{\partial X_{v}}
$$

From the theory it follows that $K$ represents the total probability per unit time for any elementary event to occur at all, and relation (4) implies that the irreversible process can be described completely from the knowledge of the scalar function $K$.

It must be stressed that the fundamental relation (4) is less strong than (1); it does not represent the generalization of Onsager relations since it refers only to the unilateral flows and not to the net flows $J$. Some examples where it is possible to calculate $K$ explicitly have been considered by van Kampen, among them the asymmetric Rayleigh particle ${ }^{5}$ ). It is proved there that the net momentum flow $J_{p}$ can be obtained as the derivative of a function $K(v)$ and, consequently, that the components of this flow obey a reciprocal relation of the form

$$
\frac{\partial}{\partial v_{j}}\left(J_{p}\right)_{i}=\frac{\partial}{\partial v_{i}}\left(J_{p}\right)_{j} .
$$

Uhlenbeck has remarked ${ }^{6}$ ) that when the drag experienced by the Rayleigh particle is calculated kinetically, its components do not satisfy the previous relation. The reason for this inconsistency is that in the model considered in van Kampen's article one of the essential rulcs of the theory was not fully applied, namely, the decomposition of the total flows into a combination of unilateral flows originating in each of the partial systems. The object of this paper is to consider once more the Rayleigh particle, but using a model in which it is possible to carry out the separation of the net flows into unilateral flows explicitly. The unilateral flows obtained depend only on the state of the subsystem where they originate and we prove that they obey the fundamental relation (4); this is in fact the principal feature of the model considered, that there is a complete separation between the subsystems and of the unilateral flows originating in each of them.

\section{Description of the model}

Consider a Knudsen gas in thermodynamic equilibrium, with molecules of mass $m$, number density $n$ and moving with a total momentum $p$. A particle with mass $M(M>m)$ is immersed in it, and due to the random impacts of the molecules it acquires a macroscopic velocity $V$ with respect to the laboratory system. The shape of this Rayleigh particle is arbitrary, but it will be assumed that its 
orientation is fixed, i.e. the normal unit vector $\hat{\boldsymbol{n}}$ associated with each surface element, and by convention directed into the particle, has a fixed direction in spite of the collisions.

Let us interpret the collisions in the following manner: a gas molecule hits the Rayleigh particle and sticks to it until it is desorbed again at a random time. This is not the usual picture of Maxwell boundary conditions, and with the introduction of this random delay the instantaneous character of the collision has been destroyed. The collision has been replaced by a sequence of two independent elementary events, namely, the "adsorption" and the "desorption" of a molecule from the Rayleigh particle; with this interpretation we are really describing a particle with an absorbing surface. This model enables one to decompose the original system into two subsystems: $\mathscr{S}^{1}$ the original gas in equilibrium without the adsorbed molecules and $\mathscr{S}^{2}$, consisting of the Rayleigh particle including the adsorbed molecules. The usefulness of this decomposition hinges on the fact that the state of each subsystem can be described independently of the other, with the unilateral flows depending only on the state of the source system, as will be shown in detail in the next sections.

As state variables of $\mathscr{S}^{1}$ we choose the total number of particles $N^{1}$, the total momentum $\boldsymbol{P}^{1}$ and the total energy $E^{1}$. In order to describe $\mathscr{S}^{2}$ it is necessary to specify more carefully what is meant by the adsorption. We suppose first that the adsorbed molecules are not attached to a specific point on the surface of the Rayleigh particle, but rather, that they form a thin molecular layer surrounding the particle. Within this layer there are no preferred positions, so that a molecule has the same probability to be at any point. We consider this layer as an ideal gas in equilibrium with the Rayleigh particle, and thus, $\mathscr{S}^{2}$ can also be described by its total number of particles $N^{2}$, the total momentum

$$
\boldsymbol{P}^{2}=\left(M+m N^{2}\right) \boldsymbol{V}^{2}
$$

and the total energy $E^{2}$ (the superscript 2 indicates that the variable is attached to subsystem $\mathscr{S}^{2}$ ). According to the above interpretation each time a molecule hits the Rayleigh particle, it transfers mass, momentum, and energy from $\mathscr{S}^{1}$ to $\mathscr{S}^{2}$, giving rise to the unilateral flows $F_{v}{ }^{11}$. Similarly, when later the molecule is desorbed it carries off its mass, momentum and energy, contributing then to the opposite unilateral flows $F_{v}^{12}$. Thus, the transfers for each elementary event are given by

$$
\Delta N^{i}=1, \quad \Delta \boldsymbol{P}^{i}=m v, \quad \Delta E^{i}=\frac{1}{2} m v^{2}, \quad i=1,2,
$$

with $v$ being also measured with respect to the laboratory system. 
3. Unilateral flows from $\mathscr{S}^{1}$ to $\mathscr{S}^{2}$

Since $\mathscr{S}^{1}$ is an ideal gas with definite momentum and energy, its entropy as a function of its state variables is given by:

$$
\begin{aligned}
S^{1}\left(N^{1}, \boldsymbol{P}^{1}, E^{1}\right)= & -\frac{3}{2} N^{1} \log N^{1}+N^{1} \log V / N^{1} \\
& +\frac{3}{2} N^{1} \log \left[E^{1}-\frac{\left(\boldsymbol{P}^{1}\right)^{2}}{2 m N^{1}}\right]+c N^{1},
\end{aligned}
$$

where $c$ is a constant and $V$ the volume occupied by $\mathscr{S}^{1}$.

It is useful to describe the system by means of the generalized forces defined by:

$$
X_{v}^{r}=\frac{\partial S(a)}{\partial a_{v}^{r}},
$$

where $a_{v}^{r}$ stands for the state variables $N, \boldsymbol{P}, E$ of subsystem $r$. The temperature $T$ is related to the energy by

$$
E^{1}-\frac{\left(\boldsymbol{P}^{1}\right)^{2}}{2 m N^{1}}=\frac{3}{2} N^{1} k T^{1}
$$

We now evaluate the derivatives (8) explicitly, using $T^{1}$ as an abbreviation defined by (9)

$$
\begin{aligned}
& X_{N}^{1}=\frac{\left(\boldsymbol{P}^{1}\right)^{2}}{2 m\left(N^{1}\right)^{2}} \frac{1}{k T^{1}}+\log \left[\frac{V}{N^{1}}\left(k T^{1}\right)^{3 / 2}\right], \\
& X_{P}^{1}=-\frac{\boldsymbol{P}^{1}}{m N^{1}} \frac{1}{k T^{1}} \\
& X_{E}^{1}=\frac{1}{k T^{1}} .
\end{aligned}
$$

Inverting these equations we obtain the state variables of $\mathscr{S}^{1}$ in terms of the generalized forces

$$
\begin{aligned}
& \frac{N^{1}}{V}=\left(X_{E}^{1}\right)^{-3 / 2} \exp \left[-X_{N}^{1}+\frac{m}{2} \frac{\left(X_{P}^{1}\right)^{2}}{X_{E}^{1}}\right], \\
& \frac{P^{1}}{N^{1}}=-m \frac{X_{P}^{1}}{X_{E}^{1}} \\
& \frac{E^{1}}{N^{1}}=\frac{3}{2} \frac{1}{X_{E}^{1}}+\frac{m}{2}\left(\frac{X_{P}^{1}}{X_{E}^{1}}\right)^{2} .
\end{aligned}
$$


A molecule can collide with a surface element $\mathrm{d} O$ of the Rayleigh particle only when its velocity obeys the following condition:

$$
\hat{n} \cdot\left(v-V^{2}\right)>0 .
$$

Consequently, the probability that a molecule with velocity between $v$ and $v+\mathrm{d} v$ will hit the Rayleigh particle is given by

$$
P\left(\hat{\boldsymbol{n}}, \boldsymbol{v}, \boldsymbol{V}^{2} ; N^{1}, P^{1}, T^{1}\right) \mathrm{d} \boldsymbol{v}=\hat{\boldsymbol{n}} \cdot\left(\boldsymbol{v}-V^{2}\right) f\left(\boldsymbol{v}, N^{1}, P^{1}, T^{1}\right) \mathrm{d} \boldsymbol{v},
$$

where $f$ is the Maxwell-Boltzmann velocity distribution of the gas molecules in $\mathscr{S}^{1}$

$$
f\left(v, N^{1}, P^{1}, T^{1}\right)=\frac{N^{1}}{V}\left(\frac{m}{2 \pi k T^{1}}\right)^{3 / 2} \exp \left[\frac{-m}{2 k T^{1}}\left(v-\frac{\boldsymbol{P}^{1}}{m N^{1}}\right)^{2}\right] .
$$

With (13), (14), (15), this distribution can be expressed in terms of the generalized forces, so that

$$
\begin{aligned}
& P\left(\hat{\boldsymbol{n}}, \boldsymbol{v}, \boldsymbol{V}^{2} ; X_{N}^{1}, X_{P}^{1}, X_{E}^{1}\right) \mathrm{d} \boldsymbol{v} \\
& \quad=(m / 2 \pi)^{3 / 2} \hat{\boldsymbol{n}} \cdot\left(\boldsymbol{v}-V^{2}\right) \exp \left[-X_{N}^{1}-m v \cdot X_{P}^{1}-\frac{m}{2} v^{2} X_{E}^{1}\right] \mathrm{d} \boldsymbol{v} .
\end{aligned}
$$

Observe that this probability depends on $V^{2}$, but this is a variable attached to the receiving system $\mathscr{S}^{2}$ and should therefore, be treated as a parameter. In terms of this probability the unilateral transfer flows from $\mathscr{S}^{1}$ to $\mathscr{S}^{2}$ are

$$
\begin{aligned}
& F_{N}^{21}=\int_{s} \mathrm{~d} 0 \int_{\hat{n} \cdot\left(v-V^{2}\right)>0} P\left(\hat{n}, v, V^{2} ; X_{N}^{1}, X_{P}^{1}, X_{E}^{1}\right) \mathrm{d} v, \\
& F_{P}^{21}=\int_{s} \mathrm{~d} 0 \int_{\hat{n} \cdot\left(v-V^{2}\right)>0} m v P\left(\hat{n}, v, V^{2} ; X_{N}^{1}, X_{P}^{1}, X_{E}^{1}\right) \mathrm{d} v, \\
& F_{E}^{21}=\int_{s} \mathrm{~d} 0 \underset{\hat{n} \cdot\left(v-V^{2}\right)>0}{\int_{2} m v^{2} P\left(\hat{n}, v, V^{2} ; X_{N}^{1}, X_{P}^{1}, X_{E}^{1}\right) \mathrm{d} v .}
\end{aligned}
$$

Eq. (19) represents the total probability for a collision to take place, that is, the total probability per unit time for an elementary event to occur at all. But according to the theory of van Kampen this is precisely the definition of the function $K^{r s}$, and we may thus identify

$$
K^{21}=F_{N}^{21}
$$


Hence, using (18) we have

$$
\begin{aligned}
K^{21}\left(V^{2} ; X_{N}^{1}, X_{P}^{1}, X_{E}^{1}\right)= & (m / 2 \pi)^{3 / 2} \int_{s} \mathrm{~d} 0 \int_{\hat{n} \cdot\left(v-V^{2}\right)>0} \hat{n} \cdot\left(v-V^{2}\right) \\
& \times \exp \left[-X_{N}^{1}-m v \cdot X_{P}^{1}-\frac{m}{2} v^{2} X_{E}^{1}\right] \mathrm{d} \boldsymbol{v} .
\end{aligned}
$$

Remembering that $V^{2}$ is only a parameter and thus is not to be differentiated, it is straightforward from this equation to check that the fundamental relation (4) is true, i.e.

$$
\begin{aligned}
& \frac{\partial K^{21}}{\partial X_{N}^{1}}=-F_{N}^{21}, \\
& \frac{\partial K^{21}}{\partial X_{P}^{1}}=-F_{P}^{21}, \\
& \frac{\partial K^{21}}{\partial X_{E}^{1}}=-F_{E}^{21} .
\end{aligned}
$$

\section{Unilateral flows from $\mathscr{S}^{2}$ to $\mathscr{S}^{1}$}

Assume that the specific heat $c_{w}$ of the Rayleigh particle itself is constant. Then the total energy of $\mathscr{S}^{2}$ is

$$
E^{2}=c_{v} T^{2}+\frac{3}{2} N^{2} k T^{2}+\cdots \frac{\left(\boldsymbol{P}^{2}\right)^{2}}{2\left(M+m N^{2}\right)} .
$$

The entropy of $\mathscr{S}^{2}$ should be the entropy of the ideal gas formed by the adsorbed molecules plus the entropy of the Rayleigh particle itself. Using (27) we get

$$
\begin{aligned}
S^{2}\left(N^{2}, \boldsymbol{P}^{2}, E^{2}\right)= & N^{2} \log \frac{W}{N^{2}} \\
& +\left(c_{v}+\frac{3 N^{2}}{2}\right) \log \frac{E^{2}-\left(\boldsymbol{P}^{2}\right)^{2} / 2\left(M+m N^{2}\right)}{c_{v}+\frac{3}{2} N^{2}},
\end{aligned}
$$

where $W$ is the volume occupied by the adsorbed molecules. The generalized forces are then

$$
X_{N}^{2}=\frac{m\left(\boldsymbol{P}^{2}\right)^{2}}{2\left(M+m N^{2}\right)^{2}} \frac{1}{k T^{2}}+\frac{3}{2} \log k T^{2}+\log \frac{W}{N^{2}}-\frac{5}{2},
$$




$$
\begin{aligned}
& X_{P}^{2}=-\frac{\boldsymbol{P}^{2}}{M+m N^{2}} \frac{1}{k T^{2}}, \\
& X_{E}^{2}=\frac{1}{k T^{2}},
\end{aligned}
$$

where we have again used $k T^{2}$ as an abbreviation defined by (27). From these relations we obtain the state variables in terms of the generalized forces;

$$
\begin{aligned}
& \frac{N^{2}}{W}=\left(X_{E}^{2}\right)^{-3 / 2} \exp \left[-X_{N}^{2}+\frac{m}{2} \frac{\left(X_{P}^{2}\right)^{2}}{X_{E}^{2}}-\frac{5}{2}\right], \\
& \frac{\boldsymbol{P}^{2}}{M+m N^{2}}=-\frac{X_{\boldsymbol{P}}^{2}}{X_{E}^{2}} \quad \text { or } \quad V^{2}=-\frac{X_{P}^{2}}{X_{E}^{2}}, \\
& E^{2}=\left(\frac{c_{v}}{k}+\frac{3}{2} N^{2}\right) \frac{1}{X_{E}^{2}}+\frac{1}{2}\left(M+m N^{2}\right)\left(\frac{X_{P}^{2}}{X_{E}^{2}}\right)^{2} .
\end{aligned}
$$

In this case a molecule will leave $\mathscr{S}^{2}$ if its velocity satisfies the condition

$$
\hat{n} \cdot\left(v-V^{2}\right)<0
$$

The probability for such emission with a velocity between $v$ and $v+\mathrm{d} v$ is

$$
Q\left(\hat{\boldsymbol{n}}, \boldsymbol{v}, N^{2}, \boldsymbol{P}^{2}, T^{2}\right) \mathrm{d} \boldsymbol{v}=\hat{\boldsymbol{n}} \cdot\left(\boldsymbol{v}-\boldsymbol{V}^{2}\right) f\left(\boldsymbol{v}, N^{2}, \boldsymbol{P}^{2}, T^{2}\right) \mathrm{d} \boldsymbol{v},
$$

with the distribution of velocities given by

$$
f\left(v, N^{2}, \boldsymbol{P}^{2}, T^{2}\right)=\frac{N^{2}}{W}\left(\frac{m}{2 \pi k T^{2}}\right)^{3 / 2} \exp \left[-\frac{m}{2 k T^{2}}\left(v-V^{2}\right)^{2}\right]
$$

Using (32), (33), (34) this equation can be expressed in terms of the generalized forces, and then the probability $Q$ can be written as

$$
\begin{aligned}
Q\left(\hat{n}, v, X_{N}^{2}, X_{P}^{2}, X_{E}^{2}\right) \mathrm{d} v= & \left(\frac{m}{2 \pi}\right)^{3 / 2} \hat{n} \cdot\left(v+\frac{X_{P}^{2}}{X_{E}^{2}}\right) \mathrm{e}^{-5 / 2} \\
& \times \exp \left[-X_{N}^{2}-m v \cdot X_{P}^{2}-\frac{m}{2} v^{2} X_{E}^{2}\right] \mathrm{d} v
\end{aligned}
$$

In terms of this probability the unilateral transfer flows from $\mathscr{S}^{2}$ to $\mathscr{S}^{1}$ are

$$
F_{N}^{12}=\int_{s} \mathrm{~d} 0 \int_{\hat{n} \cdot\left(v-V^{2}\right)<0} Q\left(\hat{n}, v, X_{N}^{2}, X_{P}^{2}, X_{E}^{2}\right) \mathrm{d} v,
$$




$$
\begin{aligned}
& F_{P}^{12}=\int_{s} \mathrm{~d} 0 \underset{\hat{n} \cdot\left(v-V^{2}\right)<0}{\int_{s}} m v Q\left(\hat{n}, v, X_{N}^{2}, X_{P}^{2}, X_{E}^{2}\right) \mathrm{d} v, \\
& F_{E}^{12}=\int_{s} \mathrm{~d} 0 \int_{\hat{n} \cdot\left(v-V^{2}\right)<0} \frac{1}{2} m v^{2} Q\left(\hat{n}, v, X_{N}^{2}, X_{P}^{2}, X_{E}^{2}\right) \mathrm{d} v .
\end{aligned}
$$

As before we may identify

$$
K^{12}=F_{N}^{12}
$$

and using (38), (39) we get

$$
\begin{aligned}
K^{12}\left(X_{N}^{2}, X_{P}^{2}, X_{E}^{2}\right)= & \left(\frac{m}{2 \pi}\right)^{3 / 2} \int_{s} \mathrm{~d} 0 \int_{\hat{n} \cdot\left(v+X_{\left.P^{2} / X_{E}^{2}\right)<0}\right.} \hat{\boldsymbol{n}} \cdot\left(v+\frac{X_{P}^{2}}{X_{E}^{2}}\right) \mathrm{e}^{-5 / 2} \\
& \times \exp \left[-X_{N}^{2}-m \boldsymbol{v} \cdot X_{P}^{2}-\frac{m}{2} v^{2} X_{E}^{2}\right] \mathrm{d} \boldsymbol{v} .
\end{aligned}
$$

From this equation we can obtain the fundamental relation for the unilateral flows. For the flow of particle follows immediately that

$$
\frac{\partial K^{12}}{\partial X_{N}^{2}}=-F_{N}^{12}
$$

For the remaining flows the check is less direct, because differentiation of the integrand of (43) will give two terms

$$
\begin{aligned}
\frac{\partial K^{12}}{\partial X_{P}^{2}}= & \int_{s} \mathrm{~d} 0 \int_{\hat{n} \cdot\left(v-V^{2}\right)<0} \frac{\hat{n}}{X_{E}^{2}} \exp \left[-X_{N}^{2}-m v \cdot X_{P}^{2}-\frac{m}{2} v^{2} X_{E}^{2}\right] \mathrm{d} v \mathrm{e}^{-5 / 2} \\
& -\int_{s} \mathrm{~d} 0 \int_{\hat{n} \cdot\left(v-V^{2}\right)<0} \hat{n} \cdot\left(v+\frac{X_{P}^{2}}{X_{E}^{2}}\right) m v \exp \left[-X_{N}^{2}-m v \cdot X_{P}^{2}-\frac{m}{2} v^{2} X_{E}^{2}\right] \mathrm{d} v .
\end{aligned}
$$

Since $T^{2}$ is constant within $\mathscr{S}^{2}$, using the divergence theorem it can be proved that the first term of the last expression is zero, and then

$$
\frac{\partial K^{12}}{\partial X_{P}^{2}}=-H_{P}^{12}
$$

In a similar way it can be proved that the fundamental relation (4) is also true for the flow of energy, i.e.,

$$
\frac{\partial K^{12}}{\partial X_{E}^{2}}=-F_{E}^{12}
$$




\section{Generalization}

In the model discussed above we considered the adsorbed molecules as a gas contained within a layer surrounding the Rayleigh particle where the molecules move freely. Let us now suppose that the surface of the Rayleigh particle is divided into $n$ small cells of different volumes $\omega_{i}$ and that each adsorbed molecule stays in a given cell until it is desorbed. In this way we take into account that the molecules get stuck to a particular point of the Rayleigh particle surface. In reality the mobility in the adsorbed layer varies greatly. For a monolayer, or only partial coverage of the surface, it may be small or zero but if the layer is several molecules thick it may be more like a liquid ${ }^{7-9}$ ). However that may be, we are here interested in the transport flows and not in the adsorbing properties of the layer. Assume that the $N_{i}$ molecules of cell $\delta_{i}$ form an ideal gas in equilibrium, moving as a whole with the average velocity $V^{2}$ of the Rayleigh particle. The subsystems $\mathscr{S}^{1}$ and $\mathscr{S}^{2}$ are defined in the same way as before, and the unilateral flows from $\mathscr{S}^{1}$ to $\mathscr{S}^{2}$ are still given by eqs. (19), (20), (21); but now $\mathscr{S}^{2}$ has to be described in a slightly different way. We now choose as state variables for $\mathscr{S}^{2}: N_{i}^{2}$, the occupation number of each cell; $\boldsymbol{P}^{2}$, the total momentum and the total energy $E^{2}$. The velocity $\boldsymbol{V}^{2}$ is a function of these variables

$$
V^{2}=\frac{P^{2}}{M+m \sum_{i=1}^{n} N_{i}^{2}} .
$$

Since there is no exchange of molecules between the different cells, any molecule released from a cell must go into $\mathscr{S}^{1}$ transferring its energy and momentum. This constitutes an elementary event and in this manner the unilateral flows from $\mathscr{S}^{2}$ to $\mathscr{S}^{1}$ are established. The elementary transfers are

$$
\Delta N_{i}^{2}=1, \quad \Delta \boldsymbol{P}^{2}=m \boldsymbol{v}, \quad \Delta E^{2}=\frac{1}{2} m v^{2} .
$$

From (28) we can express the entropy of each cell as

$$
\begin{aligned}
S_{i}\left(N_{i}, \boldsymbol{P}^{2}, E_{i}\right)= & N_{i} \log \frac{W_{i}}{N_{i}} \\
& +\left(c_{v}+\frac{3}{2} N_{i}\right) \log \frac{E_{i}-\left(\boldsymbol{P}^{2}\right)^{2} / 2\left(M+m N_{i}\right)}{c_{v}+\frac{3}{2} N_{i}}
\end{aligned}
$$

and consequently the entropy of subsystem $\mathscr{S}^{2}$ is

$$
\begin{aligned}
S^{2}\left(N_{i}^{2}, \boldsymbol{P}^{2}, E^{2}\right)= & \sum_{i=1}^{n} N_{i}^{2} \log \frac{W_{i}}{N_{i}^{2}} \\
& +\left(c_{v}+\frac{3}{2} \sum_{i} N_{i}^{2}\right) \log \frac{E^{2}-\left(\boldsymbol{P}^{2}\right)^{2} / 2\left(M+m \sum_{i} N_{i}^{2}\right)}{c_{v}+\frac{3}{2} \sum_{i} N_{i}^{2}} .
\end{aligned}
$$


From this the generalized forces are obtained according to (8). It is again convenient to use the temperature given by

$$
E^{2}=c_{v} T^{2}+\frac{3}{2} k T^{2} \sum_{i} N_{i}^{2}+\frac{\left(\boldsymbol{P}^{2}\right)^{2}}{2\left(M+\sum_{i} m N_{i}^{2}\right)},
$$

where $\boldsymbol{P}^{2}$ is

$$
\boldsymbol{P}^{2}=M \boldsymbol{V}^{2}+\boldsymbol{V}^{2} \sum_{i} m N_{i}^{2} .
$$

We obtain

$$
\begin{aligned}
& X_{N_{i}}^{2}=\sum \log \frac{W_{i}}{N_{i}^{2}}-\frac{3}{2}-n+\frac{3}{2} \log T^{2}+\frac{m}{2 T^{2}} \frac{\left(\boldsymbol{P}^{2}\right)^{2}}{\left(M+\sum_{i} m N_{i}^{2}\right)^{2}}, \\
& X_{P}^{2}=-\frac{1}{k T^{2}} \frac{\boldsymbol{P}^{2}}{M+\sum_{i} m N_{i}^{2}}, \\
& X_{E}^{2}=\frac{1}{k T^{2}},
\end{aligned}
$$

where $k$ is the Boltzmann constant and $n$ the total number of cells. From these equations we obtain the state variables in terms of generalized forces

$$
\begin{aligned}
& \prod_{i} \frac{N_{i}^{2}}{W_{i}}=\left(X_{E}^{2}\right)^{-3 / 2} \exp \left[-X_{N_{i}}^{2}+\frac{m}{2} \frac{\left(X_{P}^{2}\right)^{2}}{X_{E}^{2}}-\frac{3}{2}-n\right], \\
& \frac{\boldsymbol{P}^{2}}{M+\sum_{i} m N_{i}^{2}}=-\frac{X_{P}^{2}}{X_{E}^{2}}, \\
& E^{2}=\left(\frac{c_{v}}{k}+\frac{3}{2} \sum_{i} N_{i}^{2}\right) \frac{1}{X_{E}^{2}}+\frac{1}{2}\left(M+\sum_{i} m N_{i}^{2}\right)\left(\frac{X_{P}^{2}}{X_{E}^{2}}\right) .
\end{aligned}
$$

According to (38) we can write for the probability of desorption of a molecule from one of the cells

$$
\begin{aligned}
Q_{i}\left(\hat{\boldsymbol{v}}_{i}, \boldsymbol{v}, X_{N}^{2}, X_{\boldsymbol{P}}^{2}, X_{E}^{2}\right) \mathrm{d} v= & \left(\frac{m}{2 \pi}\right)^{3 / 2} \hat{\boldsymbol{v}}_{i} \cdot\left(v+\frac{X_{P}^{2}}{X_{E}^{2}}\right) \mathrm{e}^{-5 / 2} \\
& \times \exp \left[-X_{N_{i}}^{2}-m v \cdot X_{P}^{2}-\frac{m}{2} v^{2} X_{E}^{2}\right] \mathrm{d} v
\end{aligned}
$$

and consequently the transfer flows from each cell to $\mathscr{S}^{1}$ are

$$
F_{N}^{1 i}=\delta_{i} \int_{\hat{v}_{t} \cdot\left(v-\nu^{2}\right)<0} Q_{i}\left(\hat{v}_{i}, v, X_{N_{i}}^{2}, X_{P}^{2}, X_{E}^{2}\right) \mathrm{d} v,
$$




$$
\begin{aligned}
& F_{P}^{1 i}=\delta_{i} \int_{\hat{v}_{i} \cdot\left(v-V^{2}\right)<0} m v Q_{i}\left(\hat{v}_{i}, v, X_{N_{i}}^{2}, X_{P}^{2}, X_{E}^{2}\right) \mathrm{d} v, \\
& F_{E}^{1 i}=\delta_{i_{\hat{v}_{i} \cdot\left(v-V^{2}\right)<0}} \int_{\frac{1}{2} m v^{2} Q_{i}\left(\hat{v}_{i}, v, X_{N_{i}}^{2}, X_{P}^{2}, X_{E}^{2}\right) \mathrm{d} v,},
\end{aligned}
$$

where $\delta_{i}$ is the surface area of each cell. The total unilateral flows from $\mathscr{S}^{2}$ to $\mathscr{S}^{1}$ are then

$$
F_{N_{i}}^{12}=\sum_{i} F_{N}^{1 i} ; \quad F_{P}^{12}=\sum_{i} F_{P}^{1 i} ; \quad F_{E}^{12}=\sum_{i} F_{E}^{1 i}, \quad i=1, \ldots, n .
$$

As in the previous sections we may identify

$$
K^{1 i}=F_{N}^{1 i}
$$

$K^{11}$ being the probability for a molecule to be desorbed from the cell $i$. Then the total probability that molecules are desorbed from the different cells is

$$
K^{12}=\sum_{i}^{n} K^{1 i}=\sum_{i}^{n} F_{N}^{1 i}
$$

Since we know that the flows from each cell to $\mathscr{S}^{1}$ obey the fundamental relation, we have that

$$
\frac{\partial K^{12}}{\partial X_{N_{i}}}=\frac{\partial}{\partial X_{N_{i}}} \sum_{j} K^{1 j}=\sum_{i} \frac{\partial K^{1 i}}{\partial X_{N_{i}}}=-\sum_{i} F_{N_{i}}^{1 i}=-F_{N_{i}}^{12} .
$$

In a similar way for the flows of momentum and energy we find

$$
\begin{aligned}
& \frac{\partial K^{12}}{\partial X_{P}^{2}}=-F_{P}^{12}, \\
& \frac{\partial K^{12}}{\partial X_{E}^{2}}=-F_{E}^{12} .
\end{aligned}
$$

Remark. Observe that the flows of particles $F_{N}^{1 i}$ satisfy the fundamental relation when the derivatives of $K^{1 i}$ are taken with respect to the corresponding $X_{N_{i}}$, but there is no $X_{N}$ which can be associated with the complete subsystem $\mathscr{S}^{2}$ such that

$$
\frac{\partial K^{12}}{\partial X_{N}}=F_{N}^{12}
$$

whereas for the total flows of momentum and energy this is possible and is expressed by (66), (67). 


\section{Discussion}

It has been shown that the unilateral flows $F_{v}^{21}$ and $F_{v}^{12}$ obey the fundamental relation (4) and, consequently, they can be obtained from the knowledge of the scalar functions (23) and (43), respectively. The phenomenological equations are of the general form (3) and they are also determined once the unilateral flows are known. Then, in principle, the macroscopic evolution of the system is determined. The main assumption made was the particular model of the collision, which allowed us to decompose the total flows into unilateral flows that depend only on the state variables of the source system; at worst the receiving system variables may appear in the unilateral flows as parameters, but this does not affect the validity of the fundamental relation, as was shown in section 3 . The separability into subsystems and unilateral flows is the main feature of the model, which represents a simple example that can be described with the theory of van Kampen.

\section{Acknowledgements}

R.F.R. is greatly indebted to Professor N.G.van Kampen for the kind hospitality received at the Instituut voor Theoretische Fysica te Utrecht. He also acknowledges support received from the Universidad Nacional Autónoma de México under contract with the Banco de México, S.A.

\section{References}

I) G. Oster, A. Pereison and A. Katchalsky, Nature 234 (1971) 393.

2) R.K.Brayton and J.K. Moser, Quart. Appl. Math. 22 (1964) 1, 81.

3) D. G. B. Edelen, Intern. J. Eng. Sci. 10 (1972) 481.

4) N.G.van Kampen, Physica 67 (1973) 1.

5) N.G.van Kampen, Physica 67 (1973) 1, section 7;

Lord Rayleigh, Phil. Mag. 32 (1891) 424, or Scientific Papers, Cambridge 3 (1902) 473.

6) G. E. Uhlenbeck, private communication (1974).

7) I.Langmuir, J. Am. Chem. Soc, 40 (1918) 1361.

8) S. Brunauer, P.H.Emmett and E. Teller, J. Am. Chem. Soc. 60 (1932) 309.

9) S.Brunauer, L.E.Copeland and D.L.Kantro, in: The Solid-Gas Interface, Vol. 1, ed. E.A. Flood (Marcel Dekker, New York, 1967) p. 77. 